\title{
A Smart Image Processing-based System for Parking Space Vacancy Management
}

\author{
Benjamin Kommey \\ Department of Computer Engineering \\ KNUST \\ Kumasi, Ghana
}

\author{
Ernest O. Addo \\ Department of Computer Engineering \\ KNUST \\ Kumasi, Ghana
}

\author{
Andrew S. Agbemenu \\ Department of Computer Engineering \\ KNUST \\ Kumasi, Ghana
}

\begin{abstract}
Drivers often encounter problems associated with locating empty parking slots in parking areas. This paper presents a smart parking lot management system which operates using image processing. An image processing algorithm is used to detect empty parking areas from aerial images of the parking space. The algorithm processes the image, extracts occupancy information concerning spots, and their positions thereof. The system also reports if individual parking spots are occupied or otherwise. Occupancy information is made available to newly arriving drivers by projecting it unto large displays positioned at vantage points near the vicinity. The smart parking lot management system reduces the stress and time wastage associated with car parking and makes management of such areas less costly.
\end{abstract}

\section{General Terms}

Parking Space Vacancy Management, Image Processing

\section{Keywords}

Edge Detection, Morphological Dilation,WiFi, ROI, FOV

\section{INTRODUCTION}

In recent times, household vehicles have increased rapidly with population growth and economic development. Meanwhile, spaces required to park these cars in public places has rather been in short supply and are becoming very costly too [14, 6, 12]. This rising imbalance is very evident in major attraction areas that witness the influx of large numbers of people visiting in motor vehicles. Finding parking spaces in such places during events is challenging. Vehicle owners often undergo the extremely frustrating process of driving through the entire area in an unguaranteed search of available parking spots. Productive working hours are lost, fuel is expended, and carbon emissions from conventional engine vehicles increases. Management of these areas often resort to simply providing more parking spaces in order to check this problem. This effort however, compounds the existing problem as locating vacant spots become increasingly difficult when the lot is more than half filled. It is therefore quite clear that proper management of available spaces is the most suitable solution. Contrary to the conventional use of wardens, smart systems that provide readily available spot vacancy information are needed to effectively satisfy these parking demands.
In order to assist drivers in this regard, some parking guidance systems that attempt to notify the drivers of vacant spots have been designed and installed in various parking lots. A very common system features sensors and light indicators at each parking space. The indicator which is lit by default is positioned such that it can be viewed from an appreciable distance and turns off only when a vehicle occupies the spot. Drivers are able to spot vacant spaces from a distant without having to move around the whole parking lot. There are numerous variants of this basic system proposed in recent years. These systems mainly vary based on the vehicular detection mechanism employed. The methods used ranged from the use of radio frequency identification (RFID) technology [4, 2] or infrared sensors [11] to ultrasonic sensors [7]. Among these is an RFID based smart parking system prototype developed by [8] which implements an automated process of vehicular checking in and checking out for parking lots using RFID reader devices. In another system proposed by [13], the effect of the Earth's magnetic field on magnetic sensors is harnessed to determine the vacancy status of parking spaces. Although these mechanisms achieve the basic intended function of vehicular detection, their operations are often marred by environmental factors such as changes in weather conditions and/or the presence of electromagnetic interferences. Upon detection of a vehicle in the allotted space, the system require a way to inform drivers of the occupancy status of that space. In an attempt to do this, some systems implement vehicular ad hoc networks (VANETs) with the assumption that the vehicles are capable of transmitting and receiving information to and fro sensors within a certain range [15, 3, 9]. The drawback of VANET systems is that they require that vehicles have a dedicated tamper-resistant communication device: a feature that older vehicles lack. Without modification to these vehicles, the operation of VANET system is flawed. There is therefore the need for a parking guidance system that works well with all types of vehicle without modication irrespective of their types and natures. An additional factor of concern for VANET systems is the network infrastructure employed for the vehicle detection sensors. Traditional systems such as [13] have often used wired infrastructure however recent developments have suggested the use of wireless sensor networks. In these networks, all detection sensors are able to wirelessly communicate with a central controller that has information about all nodes. Sensors can be repositioned effortlessly without the need for costly reinstallations. [5] 10] used ZigBee networks in their system implementations. In the work done by [3], WiFi was employed in the broadcast vacancy reports in the infrastructure. This paper presents the design and im- 
plementation of a smart system which uses image processing methods for vehicular detection coupled with wireless network technology for monitoring parking areas.

\section{PROPOSED SYSTEM MODEL}

\subsection{Architecture of System}

The smart parking system proposed in this paper consists of three main components. These are the parking detection nodes with incorporated WiFi access points (APs) deployed within each major section of the parking facility, a wireless local area network (WLAN) integrated local base station, and a notification system for information delivery. The architecture of the proposed system is shown in Figure 1
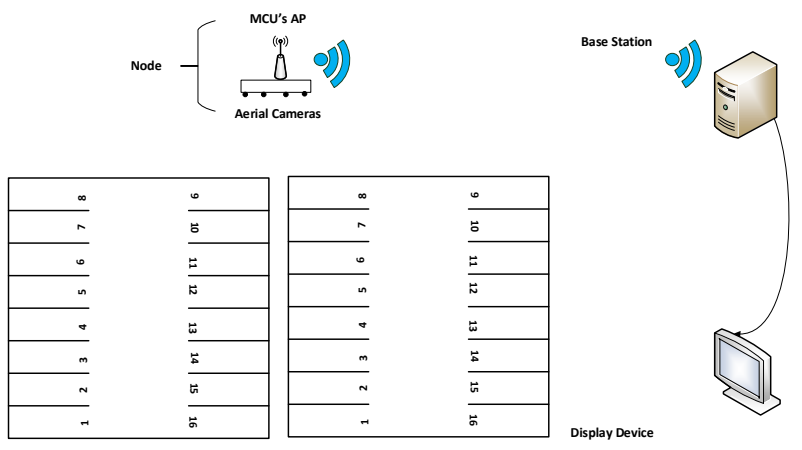

Parking Area

Fig. 1. Architecture of proposed parking management system.

The parking area is logically demarcated into major sections for easy deployment of parking detection nodes. Each major section of the area is equipped with a detection node. A node consists of wide field view cameras for taking snapshots of the assigned parking minor section at regular intervals and IEEE $802.11 \mathrm{~b} / \mathrm{g} / \mathrm{h}$ compatible microcontroller unit (MCU) for communication between the node and the base station. As shown in Figure 2, a node is affixed at a higher altitude close to the perimeter of its assigned parking section such that the node overlooks the area and the cameras are able to capture their individual regions of interest (ROIs) or minor sections in their field of view (FOV).

Each node's control unit is built around the TI CC3200 microcontroller which operates on an industry-standard ARM Cortex-M4 core running at $80 \mathrm{MHz}$. The cameras are connected to the MCU via the chip's fast parallel camera peripheral interface. The image sensor interface receives captured images from the camera immediately they are taken. The controller forwards the collected data to the base station over the setup local area network. For larger parking area, repeaters could be employed to enhance wireless communication between the nodes and the central station by regenerating signals transmitted over the network before they becomes too weak or corrupted. The base station receives the data from the nodes, processes them, retrieves vacancy information from the images, and updates the occupancy status of the parking lot and its vacancy thereof on the display positioned at vantage points.

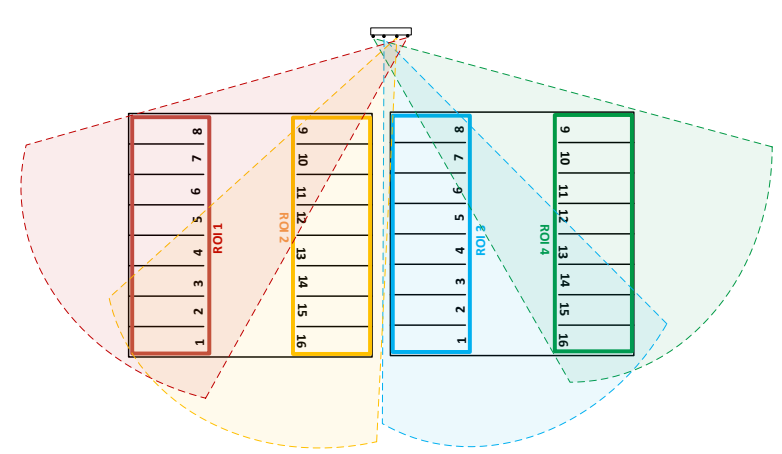

Fig. 2. Camera positions with their respective FOVs and assigned ROIs

\subsection{System Operation}

The operation of the system involved four major sub-operations as shown in Figure 3

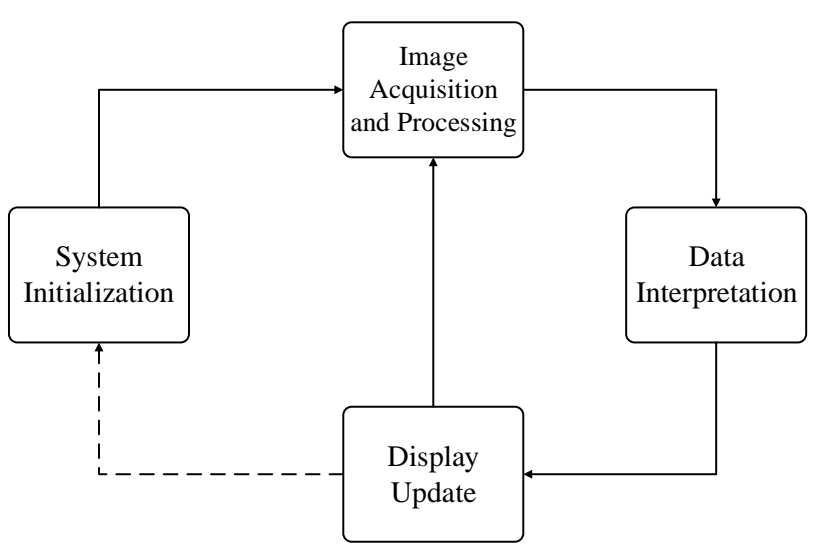

Fig. 3. Operational modules of proposed system.

These modules are system initialization, image acquisition and processing, data interpretation, and display update. The last three processes were then repeated as long as the system was active. The initialization of the parking guidance system takes place once, when the system is being set up for the first time or after a replacement of any of the systems module (shown by dashed arrow). During initialization, a refresh signal is sent from the node's controller to the image sensors in order to activate the process. The aerial cameras capture images of their assigned parking lot section whiles the area is empty. The images are transferred from the cameras to the MCU via the fast parallel image sensor interface. The MCU packages the received images into TCP packets and transmit the data from its configured on-chip Websocket (RFC 6455) HTTP server over WiFi to the base stations Websocket client application. The images are extracted from the received packets and are then processed using the image processing algorithm described in a later section of this paper. Coordinates of the marked individual parking slots and their boundaries are obtained and stored for reference 
during subsequent image processing of images. These coordinates could be used as references as long as positions and orientations of the nodes are fixed. Replacement of faulty cameras, change of positions of existing devices or installation of new ones would require re-initialization of the system thereafter. Figures 4, 5, 6, 7. and 8 illustrate the processing of the initial frame. At the base station, the received images undergo a preprocessing stage which involves the reduction of the cost of computing the image information [1]. The coloured image is grayscaled using the Luminosity method which calculates each resultant gray image pixel, $I_{\text {gray }}$ as a weighted sum of the three linear-intensity RGB values. $I_{\text {gray }}$ is related to its respective colour image RGB values: $I_{\text {red }}, I_{\text {green }}$, and $I_{\text {blue }}$ by equation 1

$$
I_{\text {gray }}=0.213\left(I_{\text {red }}\right)+0.715\left(I_{\text {green }}\right)+0.072\left(I_{\text {blue }}\right)
$$

Figure 4 shows the image of an empty minor section of a test parking area.

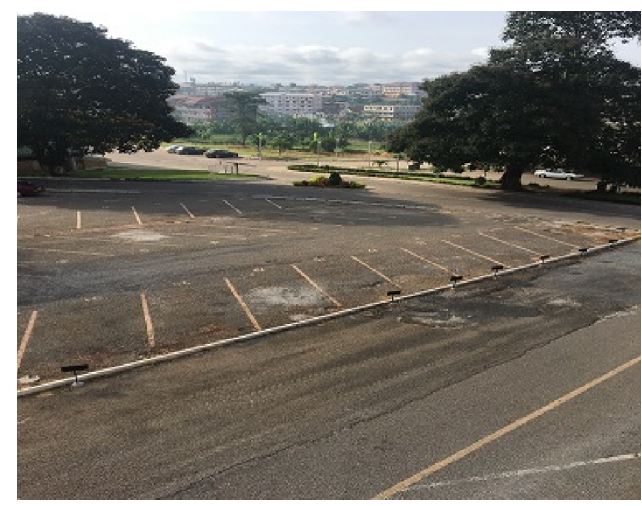

Fig. 4. An empty minor section of a test parking space.

The grayscaled image is then linearly normalized by contrast stretching to adjust image intensity values and compensate for poor image contrast which may occur due to glare. The stretched image is smoothed to remove noise and enhance accuracy of object detection and then binarized using Otsu's global thresholding method which chooses the threshold to minimize the intraclass variance of the thresholded black and white pixels. The resulting blurred and binarized image is shown in Figure 5

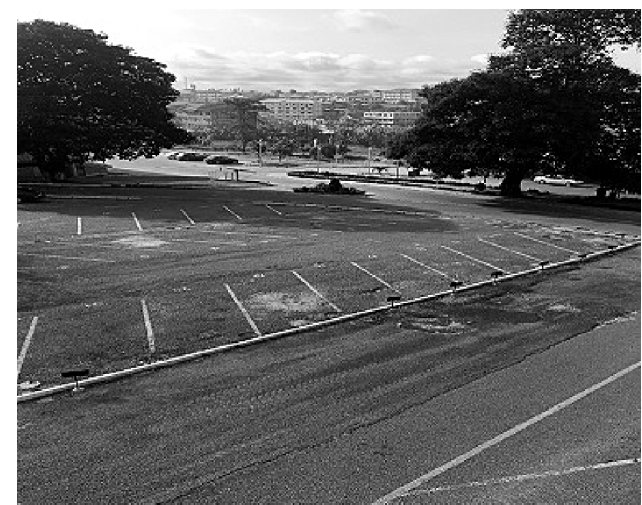

Fig. 5. Grayed, linearly normalized, and binarized image of empty minor section.
Canny edge detection is performed to identify the edges of elements in the binarized image. The edge detection process uses the first derivative of the Gaussian to approximately optimize the product of signal-to-noise ratio and localization. The Canny edge detector first smoothens the binarized image with a Gaussian filter with kernel size $(\sigma)$ of 5 to reduce noise and unwanted details and textures. The gradient is then computed using the Sobel-Feldman gradient operator. Non-maximum suppression is performed on the combined result of the Sobel-Feldman convolutions to thin out edges. Double thresholding using thresholds, $T_{1}=0.6$ and $T_{2}=0.85$ was done to ensure less noise and fewer false edges. Finally edge tracking by hysteresis was performed to determine which weak edges were actual edges. Figure 6 shows the image obtained from applying the canny edge detection algorithm to the image in Figure 5

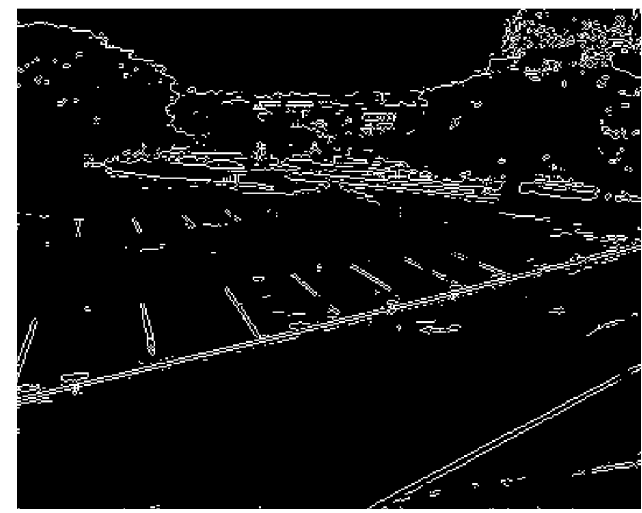

Fig. 6. Strong edges identified in binarized test image using the Canny edge detection algorithm.

A four stage morphological dilation was performed on the resulting image for better and clearer outer borders. Four different flat linear structuring elements were applied in the morphological operations. The shapes and sizes of the structuring elements $\left(S E_{n}\right)$ were:

$$
\begin{gathered}
S E_{1}=\left[\begin{array}{lll}
1 & 1 & 1
\end{array}\right] \\
S E_{2}=\left[\begin{array}{l}
1 \\
1 \\
1
\end{array}\right] \\
S E_{3}=\left[\begin{array}{lll}
1 & 0 & 0 \\
0 & 1 & 0 \\
0 & 0 & 1
\end{array}\right] \\
S E_{4}=\left[\begin{array}{lll}
0 & 0 & 1 \\
0 & 1 & 0 \\
1 & 0 & 0
\end{array}\right]
\end{gathered}
$$

The outputs of the complete dilation procedure are shown in Figure 7 A flood-fill operation is performed on background pixels of the binary image to fill holes in detected objects as shown in Figure 8 Each hole is a set of background pixels that cannot be reached by filling in the background from the edge of the image.

With the initialization of the system, the ROIs in Figures 7 and 8 are identified, marked out as shown in Figures 9 and 10 respectively. Properties of the marked regions such as coordinates of element edges and mean pixel count are stored. Figure 11 shows the 


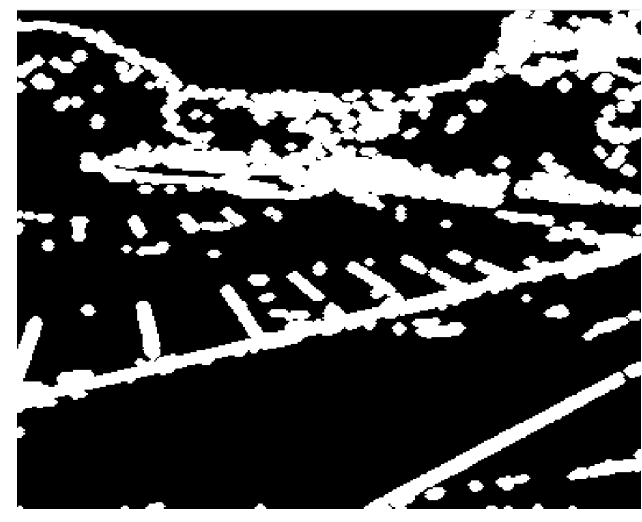

Fig. 7. Resulting edge image after fourth dilation

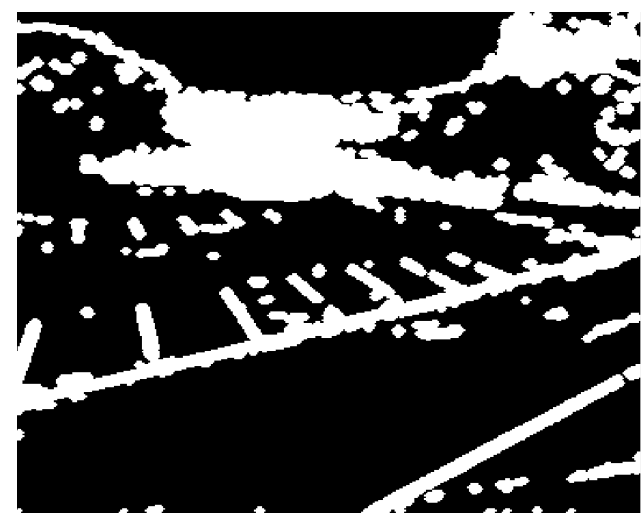

Fig. 8. Dilated edge image with holes filled

measured mean pixel value per identified spot in Figures 9 and 10 for the empty parking space during initialization.

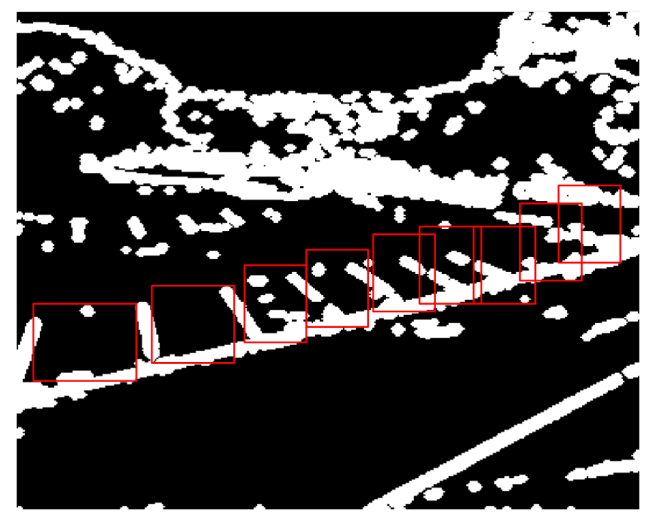

Fig. 9. Identified and marked park spaces in ROI of Figure 7

During subsequent operations of the system, parking areas in the filled binarized images are marked with bounding-boxed regions based on the coordinates obtained from the initialization process. Pixel count in each region is determined and compared against the stored initial values. Parking spots with cars would have extra edges within the marked regions which increases the mean intensity within the regions. A substantial increase in a region's pixel

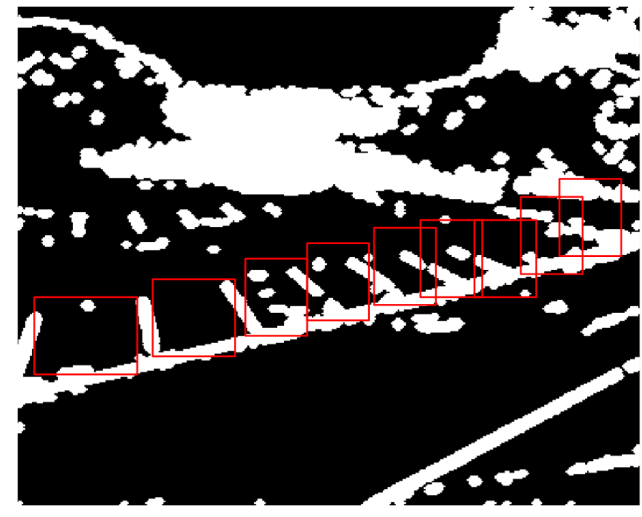

Fig. 10. Identified and marked park spaces in ROI of Figure 8

Fig. 11. A graph showing the mean intensity per empty parking region from Figures 9 and 10

count infers occupancy of the corresponding spot. This occupancy information is then sent from the base station to the display devices for use by incoming drivers.

\section{TESTING, RESULTS AND DISCUSSION}

The proposed parking space vacancy management system was tested to determine its ability to accurately extract vacancy information from captured images of the parking space. A test image was obtained using an 8MP aerially positioned camera. The image used is shown in Figure 12 The image was grayed, stretched, smoothed, and binarized by using the image processing techniques described earlier. Figure 13 shows the grayed, smoothed and binarized test image.

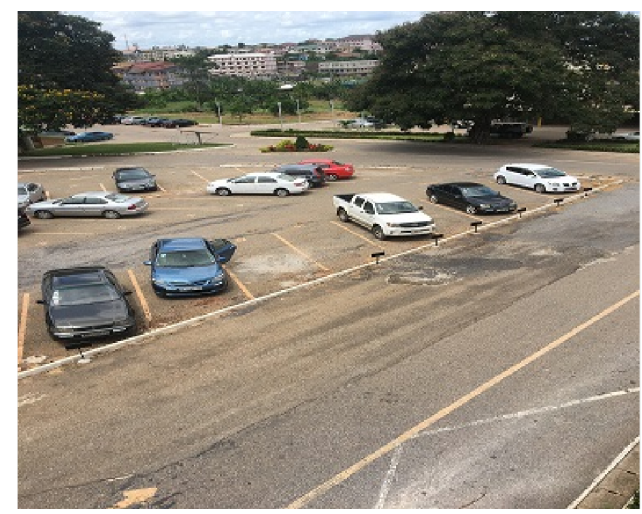

Fig. 12. Test image showing parking space minor section with cars in some spots.

The Canny edge detection algorithm was applied to identify strong edge elements in the binarized image. The obtained edge image is shown in Figure 14 The edge image was then dilated with the four stage morphological procedure using the four structural element objects stated in the previous section (Figure 15). Holes in the dilated test image were then filled. Figure 16 shows the result of the background pixel flood-filling procedure. 


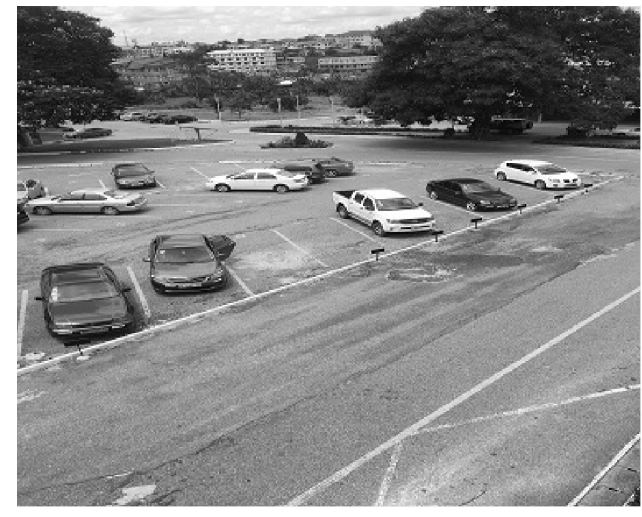

Fig. 13. Grayed, contrast stretched, and binarized test image

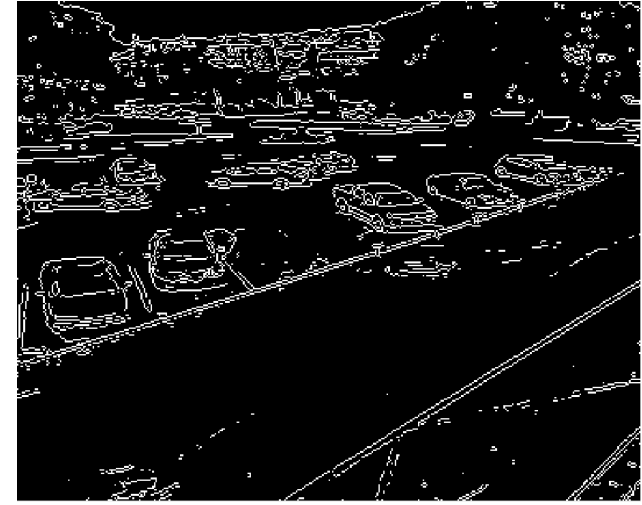

Fig. 14. Edge test image obtained using the Canny algorithm.

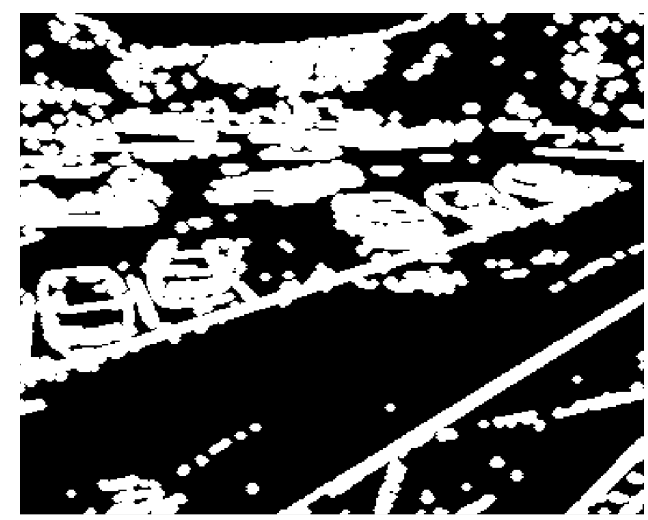

Fig. 15. Completely dilated test edge image

The parking spots in Figures 15 and 16 were all marked as shown in Figures 17 and 18 and their individual bounded pixel measurements were taken. The graph in Figure 19 shows the pixel counts for each park spots in both images

Graphical data in Figure 19 shows a substantial increase in the mean pixel count in regions which had cars occupying park spots when compared to the data in Figure 11 s graph. In spot vacancy determination, the average percentage increase in intensity recorded for occupied spots when using the filled images (Figures 10 and 18 was $166.9 \%$ with a mean absolute deviation (MAD) of 96.4 .

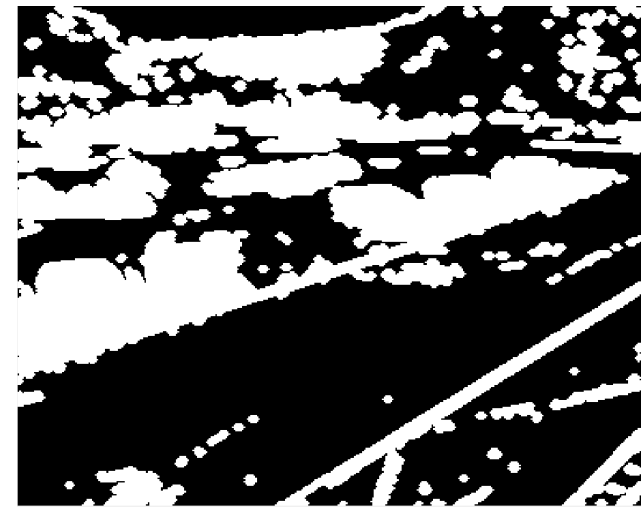

Fig. 16. Dilated edge test image with holes in detected objects filled

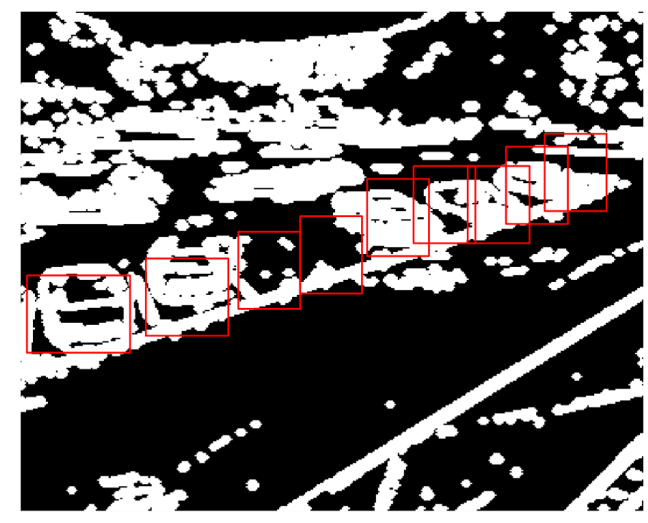

Fig. 17. Marked park spaces in dilated test edge image

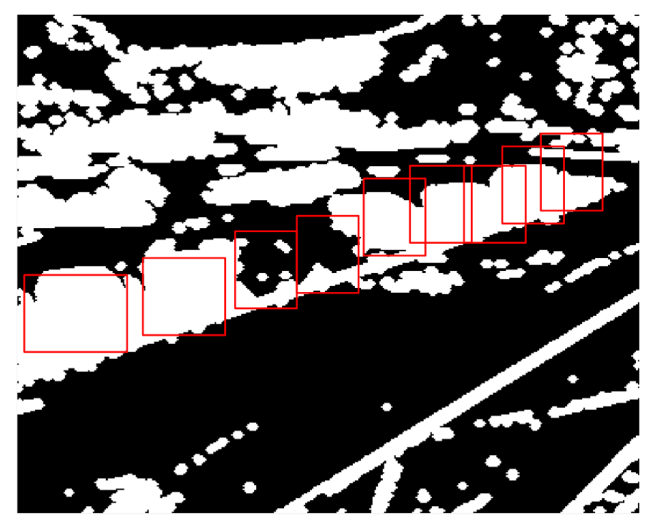

Fig. 18. Marked park slots in filled edge image

The dilated image approach recorded a mean percentage increase in pixel count was $133.7 \%$ with an MAD of 86.5 . It was observed that the use of filled edge images provided greater intensity in occupied parking regions and hence provided a much more solid basis for declaring occupancy especially in areas which lie in the middle of the camera's FOV as compared to the use of only the dilated edge images. However, due to the position and height of the camera used, the filled image approach faired averagely in detecting vacant spots (spots 6 and 8 ) located between occupied ones at the extreme 
Fig. 19. A graph showing the mean intensity per parking region from Figures 17 and 18

right side of the sensor's FOV. In these instances, the dilated edge image approach exhibited good success.

\section{CONCLUSION}

The proposed system accurately detected the presence of cars in parking slots. The filled image approach performed better than the use of dilated edge images to determine vacancy of parking spots. The two approaches could be fused into one system. The camera position could be adjusted to improve the performance. From the above set of test results, it has been shown that the proposed imageprocessing-based system is a viable option for parking space vacancy management. Other technologies such as automatic number plate recognition and traffic light control could be conjoined with this system to form holistic intelligent transport systems.

\section{REFERENCES}

[1] A. S. Agbemenu, J. Yankey, and E. O. Addo. An automatic number plate recognition system using opencv and tesseract ocr engine. International Journal of Computer Applications, 180:1-5, 2018

[2] J. P. Benson, T. O’Donovan, P. O'Sullivan, U. Roedig, and C. Sreenan. Car-park management using wireless sensor networks. In 31st IEEE Conf. Local Computer Networks, pages 588-595, 2006.

[3] M. Caliskan, D. Graupner, and M. Mauve. Decentralized discovery of free parking places. In VANET 06: 3rd international workshop on Vehicular ad hoc networks, pages 30-39, 2006.

[4] S. C. Hanche, P. Munot, P. Bagal, K. Sonawane, and P. Pooja. Automated vehicle parking system using rfid. International Journal on Research and Development, 1:89-92, 2013.

[5] Y. Hirakata, A. Nakamura, K. Ohno, and M. Itami. Navigation system using zigbee wireless sensor network for parking. In 12th International Conference on ITS Telecommunications, pages 605-609, 2012.

[6] T. Huang, Z. Zeng, and C. Li. Design and implementation of a prototype smart parking (spark) system using wireless sensor networks. In International Conference on Neural Information Processing, pages 506-515, 2012.

[7] A. Kianpisheh, N. Mustaffa, P. Limtrairut, and P. Keikhosrokiani. Smart parking system (sps) architecture using ultrasonic detector. International Journal of Software Engineering and Its Applications, 6:55-58, 2012.

[8] Z. Pala and N. Inanc. Implementation of rfid technology in parking lot access control system. In 12th International Conference on ITS Telecommunications, pages 1-3, 2007.

[9] R. Panayappan, J. M. Trivedi, A. Studer, and A. Perrig. Vanetbased approach for parking space availability. In VANET 07: Fourth ACM International Workshop on Vehicular Ad-Hoc Network, pages 75-76, 2007.

[10] S. Srikanth, P. Pramod, K. Dileep, S. Tapas, M. U. Patil, and C. B. N. Sarat. Design and implementation of a prototype smart parking (spark) system using wireless sensor networks. In International Conference on Advanced Information Networking and Applications Workshops, pages 401-406, 2009.

[11] S. Srikurinji, U. Prema, S. Sathya, and P. Manivannan. Smart parking system architecture using infrared detector. International Journal of Advanced Information and Communication Technology, 2:1113-1116, 2016.

[12] D. Teodorovic and P. Lucic. Intelligent parking systems. European Journal of Operational Research, 175:16661681, 2006.

[13] J. Wolff, T. Heuer, H. Gao, M. Weinmann, S. Voit, and U. Hartmann. Parking monitor system based on magnetic eld sensors. In IEEE Conference on Intelligent Transportation Systems, pages 1275-1279, 2006

[14] G. Yan, S. Olariu, M. C. Weigle, and M. Abuelela. Smartparking: A secure and intelligent parking system using notice. In 11th International IEEE Conference on Intelligent Transportation Systems, pages 569-574, 2008.

[15] H. Zhao, L. Lu, C. Song, and Y. Wui. Ipark: Locationaware-based intelligent parking guidance over infrastructureless vanets. International Journal of Distributed Sensor Networks, 2012:1-2, 2012 